\title{
Novel Weak Form Quadrature Element Method for Free Vibration Analysis of Hybrid Nonlocal Euler-Bernoulli Beams with General Boundary Conditions
}

\author{
Xinwei Wang \\ State Key Laboratory of Mechanics and Control of Mechanical Structures, \\ Nanjing University of Aeronautics and Astronautics, \\ Nanjing 210016, CHINA \\ *E-mail address: wangx@ nuaa.edu.cn \\ ORCID numbers of authors: \\ 0000-0003-2825-3336
}

Received date: November 2017

Accepted date: December 2017

\begin{abstract}
A novel weak form quadrature element method (QEM) is presented for free vibration analysis of hybrid nonlocal EulerBernoulli beams with general boundary conditions. For demonstrations, the stiffness and mass matrices of a beam element with Gauss-Lobatto-Legendre (GLL) nodes are explicitly given by using the nodal quadrature method together with the differential quadrature $(D Q)$ law. Convergence studies are performed and comparisons are made with exact solutions to show the excellent behavior of the proposed beam element. Case studies on hybrid nonlocal Euler-Bernoulli beams with different length scale parameters have been conducted. Accurate frequencies of the beams with different combinations of boundary conditions are obtained and presented.
\end{abstract}

Keywords: Weak form quadrature element method, hybrid nonlocal Euler-Bernoulli beam, multiple boundary conditions, free vibration.

\section{Introduction}

Due to its efficiency, the continuous mechanics approach is frequently used by researchers to study the behavior of free vibration of micro/nano-sized structures [1]. The nonlocal continuum theory [26] and the strain gradient elasticity theory [7-9] are the widely used theories of modeling the micro/ nano-scaled structures.

The hybrid nonlocal Euler-Bernoulli beam models, proposed very recently by Lim et al. [10], possess two or more independent small length-scale parameters and may model the micro/nano-structures more accurately. However, it is difficult to get analytical solutions for hybrid nonlocal EulerBernoulli beams with general boundary conditions. Therefore, numerical methods should be resorted to for solutions.

Various efficient numerical methods [4,9,11-22] can be employed to get accurate solutions of hybrid nonlocal Euler-Bernoulli beams. The discrete singular convolution (DSC) [11-14], the differential quadrature method (DQM) and differential quadrature element method (DQEM) [15-18] belong to the strong form methods. The finite element method (FEM) [4,9] and the weak form quadrature element method (QEM) [18-22] belong to the weak form methods. If the boundary conditions are not appropriately applied, the DQM may result spurious complex eigen-values [15]. Being a high order FEM, applying multiple boundary conditions by the QEM is very simple. The QEM possesses the accuracy of the global methods such as the DSC, DQM and DQEM as well as the flexibility of the 
local methods such as the FEM [20] and thus is used in present investigation. Up to dated research work related to the QEM has been well documented by Wang, Yuan and Jin [20].

The objective of this paper is to propose a novel weak form quadrature beam element for the free vibration analysis of the hybrid nonlocal Euler-Bernoulli beams. The stiffness and mass matrices of a beam element with Gauss-Lobatto-Legendre (GLL) nodes are explicitly given for the first time via the nodal quadrature method together with the differential quadrature (DQ) law. The rate of convergence of the proposed quadrature beam element is studied. Free vibration of hybrid nonlocal Euler-Bernoulli beams with different combinations of boundary conditions is analyzed. Some conclusions are drawn at the end of this paper.

\section{Higher-order nonlocal strain gradient theory}

Denote $x$ the longitudinal coordinate measured from the middle point of the hybrid nonlocal EulerBernoulli beam. Denote $I, A, E, \rho$ and $w$ the second moment of the cross-sectional area, the cross sectional area, Young's modulus, the mass density, and the transverse displacement, respectively.

For the free vibration analysis, the simplified higher-order nonlocal strain gradient theory of the hybrid nonlocal Euler-Bernoulli beam is given by [10,17]

$$
E I \frac{d^{4} w}{d x^{4}}-l^{2} E I \frac{d^{6} w}{d x^{6}}=\rho A \omega^{2} w-(e a)^{2} \rho A \omega^{2} \frac{d^{2} w}{d x^{2}}
$$

where symbols $l$ and $e a$ represent the independent length-scale parameters, and $\omega$ is the circular frequency.

The shear force, bending moment and high order bending moment are defined as $[10,17]$

$$
\begin{aligned}
& Q_{x}=E I\left(\frac{d^{3} w}{d x^{3}}-l^{2} \frac{d^{5} w}{d x^{5}}+\frac{\rho A \omega^{2}(e a)^{2}}{E I} \frac{d w}{d x}\right) \\
& M_{x}=E I\left(\frac{d^{2} w}{d x^{2}}-l^{2} \frac{d^{4} w}{d x^{4}}\right) \\
& M_{x x}=-l^{2} E I \frac{d^{3} w}{d x^{3}}
\end{aligned}
$$

To obtain the solutions by the weak form quadrature element method (QEM), the expressions of strain energy and kinetic energy are needed for derivations of the stiffness and mass matrices of the quadrature beam element. For free vibration analysis, the corresponding strain energy of the hybrid nonlocal Euler-Bernoulli beam element is given by

$$
U=\frac{E I}{2} \int_{-L / 2}^{L / 2}\left[\left(\frac{d^{2} w}{d x^{2}}\right)^{2}+l^{2}\left(\frac{d^{3} w}{d x^{3}}\right)^{2}\right] d x
$$

where symbol $L$ is the length of the beam element.

The corresponding kinetic energy of the hybrid nonlocal Euler-Bernoulli beam element is given by

$$
T=\frac{\rho A \omega^{2}}{2} \int_{-L / 2}^{L / 2}\left[(w)^{2}+(e a)^{2}\left(\frac{\partial w}{\partial x}\right)^{2}\right] d x
$$


It was pointed out early [17] that the hybrid nonlocal Euler-Bernoulli beam has totally twentyone combinations of boundary conditions. For the QEM, however, only essential boundary conditions are needed and given. Adopt the notations presented in [17], the essential boundary conditions of the hybrid nonlocal Euler-Bernoulli beam are

(a) Simply supported end-a $\left(S_{a}\right)$ :

$$
w=0, \quad \frac{d^{2} w}{d x^{2}}=0
$$

(b) Simply supported end-b $\left(S_{b}\right)$ :

$$
w=0
$$

(c) Clamped end-a $\left(C_{a}\right)$ :

$$
w=\frac{d w}{d x}=\frac{d^{2} w}{d x^{2}}=0
$$

(d) Clamped end-b $\left(C_{b}\right)$ :

$$
w=\frac{d w}{d x}=0
$$

(e) Free end-a $\left(F_{a}\right)$ :

$$
\frac{d^{2} w}{d x^{2}}=0
$$

(f) Free end-b $\left(F_{b}\right)$ : all three boundary conditions are natural ones.

\section{Formulation of the weak form quadrature beam element}

Consider an $N$-node weak form quadrature beam element. The non-dimensional coordinates of the element node are denoted by $\xi_{k}(k=1,2, \ldots, N)$, where $\xi=2 x / L$. In present investigation, GaussLobatto-Legendre (GLL) points are used as element nodes for simplicity considerations, and $\xi_{k}(k=1,2, \ldots, N)$ are the roots of the following equation,

$$
\left(1-\xi^{2}\right) \frac{d P_{N-1}(\xi)}{d \xi}=0
$$

where $P_{N-1}(\xi)$ is the $(N-1)$ th order Legendre polynomial.

The weights of nodal quadrature corresponding to integration point $\xi_{k}$, i.e., $H_{k}$, are given by 


$$
H_{k}=\frac{2}{N(N-1)} \quad(k=1, N), \quad H_{k}=\frac{2}{N(N-1)\left[P_{N-1}\left(\xi_{k}\right)\right]^{2}} \quad(k=2,3, \ldots, N-1)
$$

Since no explicit formulas exist to compute $\xi_{k}$ and $H_{k}$, the short subroutine listed in reference [20] is used to calculate the GLL points and their corresponding weights conveniently with any $N$.

The displacement function within the quadrature beam element is assumed as

$$
\begin{aligned}
w(x)= & \varphi_{1}(x) w\left(x_{1}\right)+\cdots+\varphi_{N}(x) w\left(x_{N}\right)+\left.\psi_{1}(x) \frac{d w}{d x}\right|_{x=x_{1}}+\left.\psi_{N}(x) \frac{d w}{d x}\right|_{x=x_{N}} \\
& +\left.\Gamma_{1}(x) \frac{d^{2} w}{d x^{2}}\right|_{x=x_{1}}+\left.\Gamma_{N}(x) \frac{d^{2} w}{d x^{2}}\right|_{x=x_{N}} \\
= & \sum_{j=1}^{N} \varphi_{j}(x) w_{j}+\psi_{1}(x) w_{1}^{(1)}+\psi_{N}(x) w_{N}^{(1)}+\Gamma_{1}(x) w_{1}^{(2)}+\Gamma_{N}(x) w_{N}^{(2)} \\
= & \sum_{j=1}^{N+4} h_{j}(x) \delta_{j}
\end{aligned}
$$

where the shape function $h_{j}(x)$ is also called Hermite function and its order is $(N+3)$, $\delta_{j}=w_{j}(j=1,2, \ldots, N), \delta_{N+1}=w_{1}^{(1)}, \delta_{N+2}=w_{N}^{(1)}, \delta_{N+3}=w_{1}^{(2)}, \delta_{N+4}=w_{N}^{(2)}, h_{N+1}=\psi_{1}, h_{N+2}=\psi_{N}$, $h_{N+3}=\Gamma_{1}, h_{N+4}=\Gamma_{N}$, and $h_{j}=\varphi_{j}(j=1,2, \ldots, N)$, respectively.

The $k^{\text {th }}$ order derivative of the displacement function $w(x)$ with respect to $x$ at point $i$ is given by

$$
\begin{aligned}
w^{(k)}\left(x_{i}\right) & ==\sum_{j=1}^{N+4} h_{j}^{(k)}(x) \delta_{j}=\sum_{j=1}^{N} \varphi_{j}^{(k)}\left(x_{i}\right) w\left(x_{j}\right)+\psi_{1}^{(k)}\left(x_{i}\right) w^{(1)}\left(x_{1}\right)+\psi_{N}^{(k)}\left(x_{i}\right) w^{(1)}\left(x_{N}\right) \\
& +\Gamma_{1}^{(k)}\left(x_{i}\right) w^{(2)}\left(x_{1}\right)+\Gamma_{N}^{(k)}\left(x_{i}\right) w^{(2)}\left(x_{N}\right)=\sum_{j=1}^{N+4} Q_{i j}^{(k)} \delta_{j} \quad(i=1,2, \ldots, N)
\end{aligned}
$$

where $Q_{i j}^{(k)}$ is called the weighting coefficient of the $k^{\text {th }}$ order derivative with respect to $x$. Let $l_{j}(x)$ be the Lagrange interpolation function defined by

$$
l_{j}(x)=\prod_{\substack{k=1 \\ k \neq j}}^{N} \frac{x-x_{k}}{x_{j}-x_{k}}
$$

Then the shape functions of the quadrature beam element are given by [17]

$$
\Gamma_{j}(x)=\frac{1}{2\left(x_{j}-x_{N-j+1}\right)^{2}} l_{j}(x)\left(x-x_{j}\right)^{2}\left(x-x_{N-j+1}\right)^{2} \quad(j=1, N)
$$




$$
\begin{aligned}
\psi_{j}(x)= & {\left[\frac{\left(x-x_{N-j+1}\right)}{\left(x_{j}-x_{N-j+1}\right)}\right]^{2}\left(x-x_{j}\right) l_{j}(x)-\left[2 l_{j}^{(1)}\left(x_{j}\right)+\frac{4}{x_{j}-x_{N-j+1}}\right] \Gamma_{j}(x)(j=1, N) } \\
\varphi_{j}(x)= & {\left[\frac{\left(x-x_{N-j+1}\right)}{\left(x_{j}-x_{N-j+1}\right)}\right]^{2} l_{j}(x)-\left[l_{j}^{(1)}\left(x_{j}\right)+\frac{2}{x_{j}-x_{N-j+1}}\right] \psi_{j}(x) } \\
& -\left[l_{j}^{(2)}\left(x_{j}\right)+\frac{4 l_{j}^{(1)}\left(x_{j}\right)}{x_{j}-x_{N-j+1}}+\frac{2}{\left(x_{j}-x_{N-j+1}\right)^{2}}\right] \Gamma_{j}(x) \quad(j=1, N) \\
\varphi_{j}(x)= & {\left[\frac{\left(x-x_{1}\right)\left(x-x_{N}\right)}{\left(x_{j}-x_{1}\right)\left(x_{j}-x_{N}\right)}\right]^{2} l_{j}(x) \quad(j=2,3, \cdots, N-1) }
\end{aligned}
$$

The strain energy of the beam element is obtained by substituting Eq. (14) into Eq. (5) and then integrated numerically using the nodal quadrature method, i.e., the $N$-point GLL quadrature. After doing so, $U$ is given by

$$
U=\frac{E I}{2} \sum_{k=1}^{N} \frac{L H_{k}}{2}\left\{\left(\sum_{i=1}^{N+4} Q_{k i}^{(2)} \delta_{i}\right)\left(\sum_{j=1}^{N+4} Q_{k j}^{(2)} \delta_{j}\right)+l^{2}\left(\sum_{i=1}^{N+4} Q_{k i}^{(3)} \delta_{i}\right)\left(\sum_{j=1}^{N+4} Q_{k j}^{(3)} \delta_{j}\right)\right\}
$$

Elements in the stiffness matrix of the quadrature beam element $[\hat{k}]$ are given by

$$
\hat{k}_{i j}=\frac{\partial^{2} U}{\partial \delta_{i} \partial \delta_{j}}=\sum_{k=1}^{N} \frac{L E I H_{k}}{2}\left\{Q_{k i}^{(2)} Q_{k j}^{(2)}+l^{2} Q_{k i}^{(3)} Q_{k j}^{(3)}\right\}(i, j=1,2, \ldots, N+4)
$$

By the same token, the kinetic energy of the beam element is obtained by substituting Eq. (14) into Eq. (6) and then integrated numerically using the nodal quadrature. After doing so, $T$ is given by

$$
T=\frac{\omega^{2} \rho A}{2} \sum_{k=1}^{N} \frac{L H_{k}}{2}\left\{\left(\sum_{i=1}^{N+4} h_{k i} \delta_{i}\right)\left(\sum_{j=1}^{N+4} h_{k j} \delta_{j}\right)+(e a)^{2}\left(\sum_{i=1}^{N+4} Q_{k i}^{(1)} \delta_{i}\right)\left(\sum_{j=1}^{N+4} Q_{k j}^{(1)} \delta_{j}\right)\right\}
$$

Elements in the consistent mass matrix of the quadrature beam element $[\hat{m}]$ are given by

$$
\omega^{2} \hat{m}_{i j}=\frac{\partial^{2} T}{\partial \delta_{i} \partial \delta_{j}}=\omega^{2} \sum_{k=1}^{N} \frac{L \rho A H_{k}}{2}\left\{h_{k i} h_{k j}+(e a)^{2} Q_{k i}^{(1)} Q_{k j}^{(1)}\right\} \quad(i, j=1,2, \ldots, N+4)
$$

In Eq. (23) and Eq. (24), $h_{k i}$ and $h_{k j}$ are defined as

$$
h_{k i}=h_{i}\left(x_{k}\right), h_{k j}=h_{j}\left(x_{k}\right)
$$


And $Q_{k i}^{(1)}, Q_{k j}^{(1)}, Q_{k i}^{(2)}, Q_{k j}^{(2)}, Q_{k i}^{(3)}, Q_{k j}^{(3)}$ are the weighting coefficients of the first, second and third order derivatives with respect to $x$.

The weighting coefficients of $k$-th order derivative, $Q_{i j}^{(k)}$, can be explicitly computed by [17]

$$
\begin{aligned}
& Q_{i j}^{(k)}= \Gamma_{j}^{(k)}\left(x_{i}\right)=\frac{1}{2\left(x_{j}-x_{N-j+1}\right)^{2}}\left\{l_{j}^{(k)}\left(x_{i}\right)\left(x_{i}-x_{j}\right)^{2}\left(x_{i}-x_{N-j+1}\right)^{2}\right. \\
&+2 k l_{j}^{(k-1)}\left(x_{i}\right)\left(x_{i}-x_{j}\right)\left(x_{i}-x_{N-j+1}\right)^{2}+2 k l_{j}^{(k-1)}\left(x_{i}\right)\left(x_{i}-x_{j}\right)^{2}\left(x_{i}-x_{N-j+1}\right) \\
&+4 k(k-1) l_{j}^{(k-2)}\left(x_{i}\right)\left(x_{i}-x_{j}\right)\left(x_{i}-x_{N-j+1}\right)+k(k-1) l_{j}^{(k-2)}\left(x_{i}\right)\left(x_{i}-x_{j}\right)^{2} \\
&+k(k-1) l_{j}^{(k-2)}\left(x_{i}\right)\left(x_{i}-x_{N-j+1}\right)^{2}+2 k(k-1)(k-2) l_{j}^{(k-3)}\left(x_{i}\right)\left(x_{i}-x_{N-j+1}\right) \\
&\left.+2 k(k-1)(k-2) l_{j}^{(k-3)}\left(x_{i}\right)\left(x_{i}-x_{j}\right)+k(k-1)(k-2)(k-3) l_{j}^{(k-4)}\left(x_{i}\right)\right\} \\
& Q_{i j}^{(k)}=\psi_{j}^{(k)}\left(x_{i}\right)=\left\{l_{j}^{(k)}\left(x_{i}\right)\left(x_{i}-x_{j}\right)\left(x_{i}-x_{N-j+1}\right)^{2}+k(k-1) l_{j}^{(k-2)}\left(x_{i}\right)\left[2\left(x_{i}-x_{N-j+1}\right)+\left(x_{i}-x_{j}\right)\right]\right.(i=1,2, \ldots, N ; j=1, N) \\
&\left.+k l_{j}^{(k-1)}\left(x_{i}\right)\left[\left(x_{i}-x_{N-j+1}\right)^{2}+2\left(x_{i}-x_{j}\right)\left(x_{i}-x_{N-j+1}\right)\right]+k(k-1)(k-2) l_{j}^{(k-3)}\left(x_{i}\right)\right\} /\left(x_{j}-x_{N-j+1}\right)^{2}(i=1,2, \ldots, N ; j=1, N) \\
&-\left[2 l_{j}^{(1)}\left(x_{j}\right)+\frac{4}{x_{j}-x_{N-j+1}}\right] \Gamma_{j}^{(k)}\left(x_{i}\right) \quad(i=1,2, \ldots, N ; j=2,3, \cdots, N-1) \\
& Q_{i j}^{(k)}=\varphi_{j}^{(k)}\left(x_{i}\right)=\frac{1}{\left(x_{j}-x_{1}\right)^{2}\left(x_{j}-x_{N}\right)^{2}}\left\{l_{j}^{(k)}\left(x_{i}\right)\left(x_{i}-x_{1}\right)^{2}\left(x_{i}-x_{N}\right)^{2}+2 k l_{j}^{(k-1)}\left(x_{i}\right)\left[\left(x_{i}-x_{1}\right)^{2}\left(x_{i}-x_{N}\right)\right.\right. \\
&\left.\quad+\left(x_{i}-x_{1}\right)\left(x_{i}-x_{N}\right)^{2}\right]+k(k-1) l_{j}^{(k-2)}\left(x_{i}\right)\left[\left(x_{i}-x_{1}\right)^{2}+4\left(x_{i}-x_{1}\right)\left(x_{i}-x_{N}\right)+\left(x_{i}-x_{N}\right)^{2}\right] \\
& Q_{i j}^{(k)}=\varphi_{j}^{(k)}\left(x_{i}\right)=\left\{l_{j}^{(k)}\left(x_{i}\right)\left(x_{i}-x_{N-j+1}\right)^{2}+2 k l_{j}^{(k-1)}\left(x_{i}\right)\left(x_{i}-x_{N-j+1}\right)+k(k-1) l_{j}^{(k-2)}\left(x_{i}\right)\right\} /\left(x_{j}-x_{N-j+1}\right)^{2} \\
& \quad-\left[l_{j}^{(1)}\left(x_{j}\right)+\frac{2}{x_{j}-x_{N-j+1}}\right] \psi_{j}^{(k)}\left(x_{i}\right)-\left[l_{j}^{(2)}\left(x_{j}\right)+\frac{4 l_{j}^{(1)}\left(x_{j}\right)}{x_{j}-x_{N-j+1}}+\frac{2}{\left(x_{j}-x_{N-j+1}\right)^{2}}\right] \Gamma_{j}^{(k)}\left(x_{i}\right) \\
&\left.\quad+2 k(k-1)(k-2) l_{j}^{(k-3)}\left(x_{i}\right)\left[\left(x_{i}-x_{1}\right)+\left(x_{i}-x_{N}\right)\right]+k(k-1)(k-2)(k-3) l_{j}^{(k-4)}\left(x_{i}\right)\right\} \\
& \quad
\end{aligned}
$$

In Eqs. (26)-(29), $l_{j}^{(0)}\left(x_{i}\right)=l_{j}\left(x_{i}\right)=\delta_{i j}, l_{j}^{(n)}\left(x_{i}\right)$ should be dropped when $n<0$ since the coefficient associated with it is zero, where $n=\left(k-i_{1}\right),\left(i_{1}=1,2,3\right)$. Since nodal quadrature is used, $l_{j}^{(k)}\left(x_{i}\right)$ $(k=1,2,3)$ can be computed explicitly by using the formulas of the DQM as follows [18], 


$$
l_{j}^{(1)}\left(x_{i}\right)=\tilde{A}_{i j}=\left\{\begin{array}{ll}
\frac{1}{x_{j}-x_{i}} \prod_{\substack{m=1 \\
m \neq i, j}}^{N} \frac{x_{i}-x_{m}}{x_{j}-x_{m}} & (i \neq j) \\
\sum_{\substack{m=1 \\
m \neq i}}^{N} \frac{1}{x_{i}-x_{m}} & (i=j)
\end{array}(i, j=1,2, \ldots, N)\right.
$$

And

$$
l_{j}^{(2)}\left(x_{i}\right)=\tilde{B}_{i j}=\sum_{m=1}^{N} \tilde{A}_{i m} \tilde{A}_{m j} ; l_{j}^{(3)}\left(x_{i}\right)=\tilde{C}_{i j}=\sum_{m=1}^{N} \tilde{A}_{i m} \tilde{B}_{m j}
$$

It is easy to see that $Q_{i j}^{(k)}=0$ when $k>N+3$, since $h_{j}(x)$ is a $(N+3)$ th order polynomial.

If only one element is used to model the entire beam, the equation of motion can be symbolically written as

$$
[\hat{k}]\{\delta\}=\omega^{2}[\hat{m}]\{\delta\}
$$

Eliminating the rows and columns in $[\hat{k}]$ and $[\hat{m}]$ in Eq. (32) which correspond to the zero displacements (i.e., the essential boundary conditions) yields

$$
[\bar{k}]\{\bar{\delta}\}=\omega^{2}[\bar{m}]\{\bar{\delta}\}
$$

Solving Eq. (33) by a generalized eigen-solver yields the frequencies of the hybrid nonlocal EulerBernoulli beam.

If more beam elements are used to model the entire beam structure, the assemblage procedures are exactly the same as the ones of the finite element method [20].

It is worth notice that the problem of spurious complex eigen-values will never occur since both stiffness matrix and mass matrix are symmetric matrices. It is also worth notice that both stiffness and mass matrices are not fully integrated, since the nodal quadrature is only exact for a polynomial of $(2 N-3)$ th degrees and less. If fully integrated stiffness and mass matrices are required, then Gauss quadrature should be used for efficiency considerations and $Q_{i j}^{(k)}(k=1,2,3)$ at Gauss integration points should be computed differently. More precisely, $l_{j}^{(k)}\left(x_{i}\right)$ in Eqs. (26)-(29) should be computed differently since the DQ law cannot be directly employed. The method proposed by author's group [19-22] should be used to compute $Q_{i j}^{(k)}(k=1,2,3)$ explicitly and conveniently.

\section{Results and discussion}

For convenience in presentation, three non-dimensional parameters are introduced, i.e., $\zeta=l / L$, $\tau=e a / L$ and $\lambda=\sqrt{\rho A \omega^{2} / E I}$. One $N$-node element is used to model the entire beam for simplicity.

For the $S_{a}-S_{a}$ beam, the exact solution of the frequency parameter can be easily obtained by using Navier solution method [17]. The exact non-dimensional frequency parameter is given by

$$
\lambda=\frac{n^{2} \pi^{2} \sqrt{1+\zeta^{2} n^{2} \pi^{2}}}{\sqrt{1+\tau^{2} n^{2} \pi^{2}}}(n=1,2, \ldots)
$$


where $n$ is the mode number.

Convergence studies are performed first. The number of nodes $N$ varies from 7 to 19. Figure 1 shows the convergence rate of the proposed element. The $S_{a}-S_{a}$ beam with $\zeta=0.16$ and $\tau=0.1$ is considered. The absolute differences between the QEM data and analytical solutions are presented. It is seen that the proposed QEM exhibits the exponential rate of convergence.

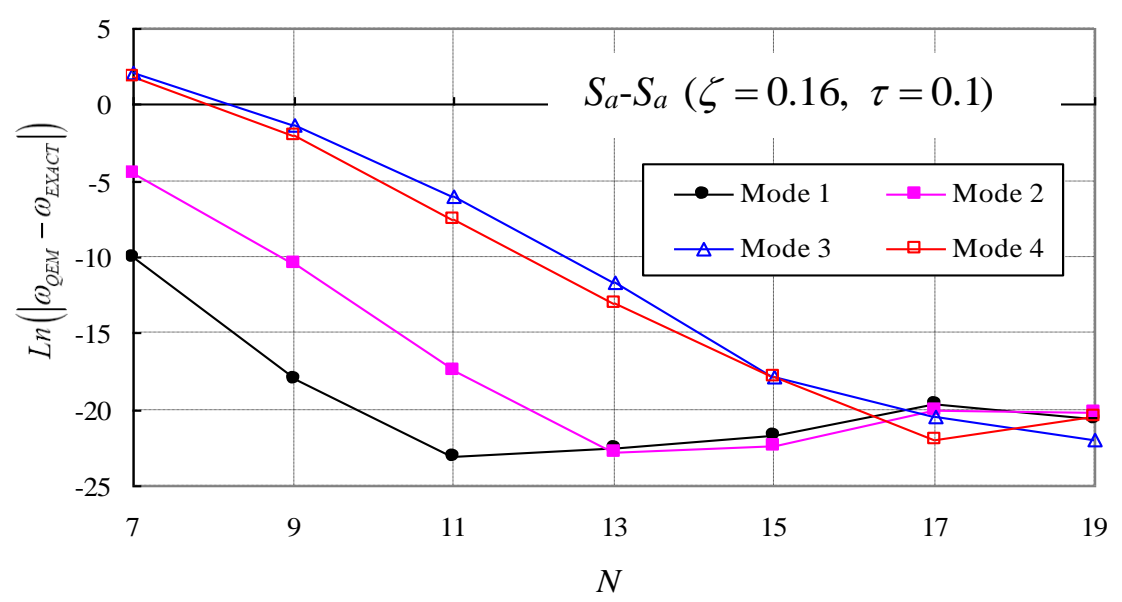

Fig. 1. Convergence rate of the proposed beam element

To demonstrate the high accuracy of the QEM further, the $S_{b}-S_{b}$ beam with $\zeta=0.2$ and $\tau=0.14$ is analyzed. Since analytical solution is not available, thus the results obtained by the DQEM [17] with $N=119$ are served as the reference data. Figure 2 shows the variation of the first five frequency parameters with the number of nodes. It is seen that the rate of convergence is high and the QEM with $N=13$ can yield accurate first five frequency parameters. It was reported early [17] that the DQEM with $N=13$ can yield accurate first five frequency parameters for the hybrid nonlocal EulerBernoulli beam. This indicates that the QEM possesses the same accuracy of the DQEM.

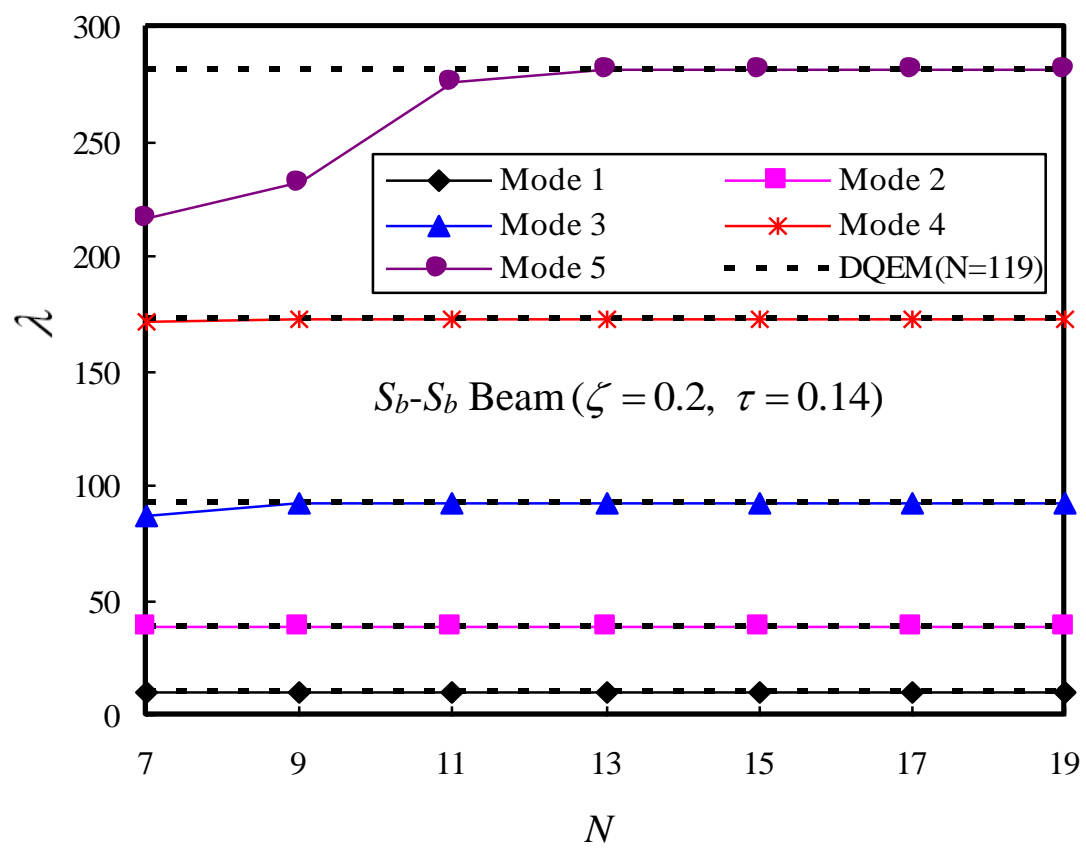

Fig. 2. Convergence of the first five frequency parameters 
The variations of the first six frequency parameters of a $C_{a}-F_{a}$ beam with one of the small lengthscale parameter are shown in Fig. 3. One of the two small length-scale parameters is fixed and the other varies from 0.008 to 0.2 .

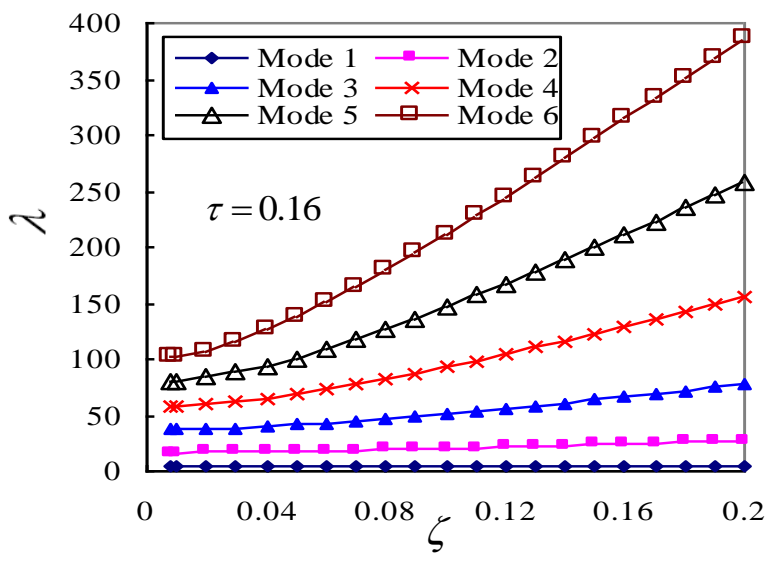

(a)

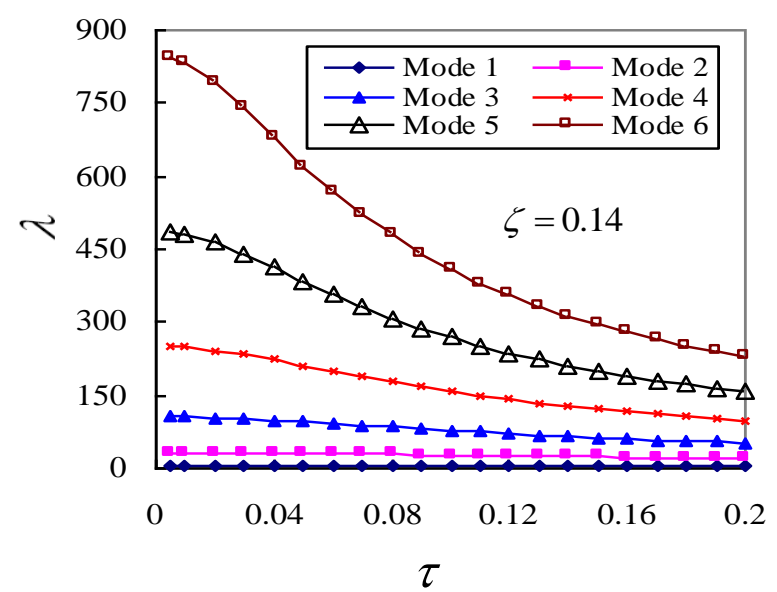

(b)

Fig.3. Effect of small length-scale parameters on the frequencies of the $C_{a}-F_{a}$ beam

To ensure the solution accuracy, the QEM results are obtained by using one 19-node beam element. It is clearly seen that the first six frequency parameters increase with the increase of $\zeta$ but decreases with the increase of $\tau$, and the effect of $\tau$ and $\zeta$ on frequencies is appreciated and opposite. The influence degree is quite different and also increases with the increase of mode numbers. It is easy to get accurate frequencies for beams with other boundary conditions and small length-scale parameter by the proposed QEM. Results are omitted to save the space.

\section{Conclusions}

A novel weak form quadrature element method is presented for free vibration analysis of hybrid nonlocal Euler-Bernoulli beams. Explicit formulas to compute the element stiffness and mass matrices are given via nodal quadrature and the DQ law. It is shown that the QEM has exponential rate of convergence. Numerical results show that the proposed QEM is computationally efficient and capable of obtaining accurate solutions of the hybrid nonlocal Euler-Bernoulli beams with any combination of boundary conditions and length-scale parameters. It is seen that the effect of the two length-scale parameters on the frequency of the beam is opposite. It is demonstrated that the QEM possesses advantages of the flexibility of the FEM and high accuracy of the DQEM. Present research extends the application range of the weak form quadrature element method.

\section{Acknowledgment}

The author acknowledges the support from the Priority Academic Program Development of Jiangsu Higher Education Institutions. 


\section{References}

[1] Behera, L., Chakraverty, S., Recent researches on nonlocal elasticity theory in the vibration of carbon nanotubes using beam models: A review. Archives of Computational Methods in Engineering. 24(3), 481-494, 2017.

[2] Eringen, A.C., On differential equations of nonlocal elasticity and solutions of screw dislocation and surface waves. Journal of Applied Physics, 54 (9), 4703-4710, 1983.

[3] Civalek, O., Demir, C., A simple mathematical model of microtubules surrounded by an elastic matrix by nonlocal finite element method. Applied Mathematics and Computation, 289, 335-352, 2016.

[4] Demir, C., Civalek, O., A new nonlocal FEM via Hermitian cubic shape functions for thermal vibration of nano beams surrounded by an elastic matrix. Composite Structures, 168, 872-884, 2017.

[5] Civalek, O., Demir, C., Bending analysis of microtubules using nonlocal Euler-Bernoulli beam theory. Applied Mathematical Modeling, 35, 2053-2067, 2011.

[6] Mercan, K., Civalek, O., Comparison of small scale effect theories for buckling analysis of nanobeams. International Journal of Engineering \& Applied Sciences, 9(3), 87-97, 2017.

[7] Mindlin, R.D., Micro-structure in linear elasticity. Archive for Rational Mechanics and Analysis, $16(1), 51-78,1964$.

[8] Akgöz, B., Civalek, O., Strain gradient elasticity and modified couple stress models for buckling analysis of axially loaded micro-scaled beams. International Journal of Engineering Sciences, 49, 1268-1280, 2011.

[9] Xu, W., Wang, L.F., Jiang, J.N., Strain gradient finite element analysis on the vibration of double-layered graphene sheets. International Journal of Computational Methods, 13, 1650011, 2016.

[10] Lim, C.W., Zhang, G., Reddy, J.N.. A higher-order nonlocal elasticity and strain gradient theory and its applications in wave propagation. Journal of the Mechanics and Physics of Solids, 78, 298-313, 2015.

[11] Wei, G.W., Discrete singular convolution for the solution of the Fokker-Planck equations. Journal of Chemical Physics, 110, 8930-8942, 1999.

[12] Wei, G.W., A new algorithm for solving some mechanical problems. Computer Methods in Applied Mechanics and Engineering, 190, 2017-2030, 2001.

[13] Wang, X., Duan, G., Discrete singular convolution element method for static , buckling and free vibration analysis of beam structures. Applied Mathematics and Computation, 234, 36-51, 2014. 
[14] Wang, X., Yuan, Z., Discrete singular convolution and Taylor series expansion method for free vibration analysis of beams and rectangular plates with free boundaries. International Journal of Mechanical Sciences, 122, 184-191, 2017.

[15] Ng, C.H.W., Zhao, Y.B., Xiang, Y., Wei, G.W., On the accuracy and stability of a variety of differential quadrature formulations for the vibration analysis of beams. International Journal of Engineering \& Applied Sciences, 1(4), 1-25, 2009.

[16] Tornabene, F., Fantuzzi, N., Ubertini, F., Viola, E., Strong formulation finite element method: A survey. Applied Mechanics Reviews, 67, 020801,2015.

[17] Wang, X., Novel differential quadrature element method for vibration analysis of hybrid nonlocal Euler-Bernoulli beams. Applied Mathematics Letters, 77, 94-100, 2018.

[18] Wang X. Differential Quadrature and Differential Quadrature Based Element Methods: Theory and Applications. Oxford: Butterworth-Heinemann, 2015.

[19] Jin, C., Wang, X., Ge, L., Novel weak form quadrature element method with expanded Chebyshev nodes. Applied Mathematics Letters, 34, 51-59, 2014.

[20] Wang, X., Yuan, Z., Jin, C., Weak form quadrature element method and its applications in science and engineering: A state-of-the-art review. Applied Mechanics Reviews, 69, 030801, 2017.

[21] Jin, C., Wang, X., Accurate free vibration of functionally graded skew plates. Transactions of Nanjing University of Aeronautics \& Astronautics, 34(2), 188-194, 2017.

[22] Jin, C., Wang, X., Weak form quadrature element method for accurate free vibration analysis of thin skew plates. Computers and Mathematics with Applications, 70, 2074-2086, 2015. 